\title{
A rapid cooperation-differentiated medium access control protocol with packet piggyback for multihop wireless networks
}

\author{
Yayan $\mathrm{Li}^{1,2}$, Kai Liư ${ }^{1 *}$, Feng Liu ${ }^{1}$ and Zhen $\mathrm{Xu}^{1}$
}

\begin{abstract}
To improve the efficiency of node cooperation and multiple access performance in multihop wireless networks, a rapid cooperation-differentiated medium access control (MAC) protocol is proposed. In the protocol, the helper selection process is divided into a priority differentiation phase and contention resolution phase for helpers with the same priority. A higher priority helper can choose an earlier minislot in the priority differentiation phase to send a busy tone. As a result, the protocol promptly selects all the highest priority helpers. The contention resolution phase for the same priority helpers can use any existing collision resolution scheme, such as the $k$-round elimination contention scheme. Helpers sending a busy tone first can proceed to the next round, while others, sensing the busy tone, subsequently withdraw from contention. Therefore, a unique optimal helper is selected from the highest priority helpers with high probability. A packet piggyback mechanism is also adopted, which allows a high data rate helper with its own data packets, to transmit these to their recipient without reservation. This significantly decreases the reservation overhead and effectively improves cooperation efficiency and channel utilization. Simulation results show that the maximum throughput of the proposed protocol is 39.6\% and $9.6 \%$ higher than those of the cooperative MAC-aggregation (CoopMACA) and instantaneous relay-based cooperative MAC protocols, respectively, in a wireless local area network environment, and $40.8 \%$ and 31.9\% higher, respectively, in an ad hoc network environment.
\end{abstract}

Keywords: Multihop wireless networks; Medium access control; Cooperative communication; Helper selection; Priority differentiation; Contention resolution; Packet piggyback

\section{Introduction}

In multihop wireless networks, signal fading in the data transmission process and signal interference among users or nodes have a significant impact on the quality of signal reception and system capacity. Cooperative communication techniques offer a solution to these challenges $[1,2]$. Such techniques use the broadcast nature of wireless communication to engage some nodes within the communication range of the sender to act as helpers in assisting the sender to transmit a data packet to its recipient, thereby effectively combating signal fading and improving spectrum efficiency and communication reliability [3,4]. These techniques are gradually being used in typical wireless

\footnotetext{
* Correspondence: liuk@buaa.edu.cn

${ }^{1}$ School of Electronics and Information Engineering, Beihang University, Beijing 100191, China

Full list of author information is available at the end of the article
}

networks, such as next-generation networks, wireless local area networks (WLANs), wireless sensor networks, and mobile ad hoc networks.

Early research work on cooperative communication techniques mainly focused on the physical (PHY) layer. It is generally assumed that a recipient can receive signals from the sender and multiple helpers either simultaneously using multiple orthogonal channels or a space-time coding scheme, or sequentially using different time slots. This results in a tradeoff between the cooperative diversity gain and other performance metrics, such as channel capacity, data rate, bandwidth efficiency, power efficiency, bit error rate (BER), and anti-interference capability. Recently, more and more research has focused on applying a cooperative communication technique to the upper layers, such as the data link layer and network layer, to enhance cooperative transmission efficiency. In the data link layer, 
the medium access control (MAC) layer is close to the PHY layer and is used to address the channel sharing problem for multiple users or nodes [5]. A good MAC protocol with multihop fair access can achieve the upper bounds on network utilization and lower bounds on delay for multihop wireless networks [6]. Furthermore, how to design a better MAC protocol based on node cooperation is critical to further improve network performance and exploit the cooperation gain. Therefore, cooperative MAC protocols have become a hot research topic $[7,8]$.

The objectives of applying a cooperative communication technique to the MAC layer to improve multiple access performance are twofold: first, to improve the information transmission rate and throughput, and second, to enhance the link reliability and anti-interference capability [1]. The former favors node cooperation over direct transmission from a sender to its recipient. The sender needs to determine whether cooperative transmission is advantageous during reservation, i.e., whether a helper can support a higher data rate from the sender to its recipient or consume a shorter data packet transmission time. If so, node cooperation is initiated. Otherwise, the sender transmits the data packet directly to its recipient. Typical protocols are the cooperative MAC (CoopMAC) protocol [8] and the relay-enabled distributed coordination function (rDCF) protocol [9], among others. These protocols exploit higher data rate transmission from the sender to its helper and from the helper to the recipient to replace the lower data rate transmission from the sender directly to its recipient, thereby improving the equivalent data rate from the sender to its recipient and decreasing the transmission time of a data packet.

The latter objective adopts the concept of cooperative automatic repeat request (ARQ), i.e., a cooperation transmission mechanism can be used only when the recipient cannot correctly receive the data packet from its sender, and then the helper retransmits the data packet to the recipient. Typical protocols for this are the cooperative diversity MAC (CD-MAC) protocol [1], the differentiated cooperative MAC (DC-MAC) protocol [10], and the cooperative time division multiple access (TDMA) protocol [11]. In the CD-MAC protocol [1], the sender and its specified optimal helper retransmit the data packet to the recipient simultaneously if direct transmission fails. This takes advantage of the enhanced signal intensity to overcome the signal fading problem and improve the antiinterference capability. To address the problems of error bursts and the limited signal transmission distance of high data rate nodes, the DC-MAC protocol [10] adopts a cooperative ARQ mechanism and randomly chooses one of the helpers with a high enough received signal-to-noise ratio (SNR) for its transmitted signal at the recipient to retransmit the data packet. Meanwhile, it uses the negative acknowledgment scheme to differentiate data packet collisions and failure data packet reception caused by channel errors and to perform channel estimation. Therefore, the correct helper is selected to retransmit the data packet only in the case of failed data packet reception, which improves cooperation efficiency and packet transmission reliability. In [11], a cooperative TDMA protocol using dynamic slot assignment scheme is presented to guarantee the transmission reliability. Not only the source node but also the cooperative nodes with no packets to transmit have the responsibility of suggesting a time slot for cooperative retransmission of the failed packet during their respective minislot of the next signaling period (SP). The suggestion of cooperative nodes has a higher priority than that of the source node, and if a cooperative node has already suggested its time slot for the failed packet, other cooperative nodes can suggest their respective time slots for other failed packets. Then, during the data transmitting period, the source node and all cooperative nodes cooperatively retransmit the failed packet in the suggested time slot, and the source node can still transmit its next packet in its own time slot. This protocol can schedule multiple packet retransmissions during multiple idle time slots in each TDMA frame, respectively.

Currently, more and more cooperative MAC protocols consider the above two aspects, including the space-time coding cooperative MAC (STiCMAC) protocol [12], the instantaneous relay-based cooperative MAC (IrcMAC) protocol [13], and the optimal relay selection MAC (ORSMAC) protocol [14]. In the STiCMAC protocol [12], multiple helpers adopt a randomly distributed space-time coding (STC) mechanism to cooperatively transmit a data packet at the same time, thereby combating serious signal fading. This protocol also uses proper STC modulation and a channel coding rate to increase the data transmission rate. In the IrcMAC protocol [13], each helper estimates the highest data rate that it can support between itself and the sender and recipient, and chooses the corresponding minislot during the relay response period to send a 1-bit signal. According to the position of the 1-bit signal, the sender selects the two best helpers for the first cooperative relay transmission and the second backup relay transmission. In the event that the first cooperative transmission fails, the backup helper relays the data packet to the recipient instead of having the sender retransmit it. This improves throughput while guaranteeing transmission reliability. From the constructed Coop-table similar to that in [8], the ORS-MAC protocol [14] selects the best relay with the maximum throughput by considering transmission failure probability for cooperative transmission.

As mentioned above, to obtain better cooperation gain, it is necessary to solve the problems of how to select helpers and how to cooperate. To address these problems, we propose a rapid cooperation-differentiated MAC (RCD-MAC) protocol for multihop wireless 
networks. According to the data rates from a helper to the sender and from the helper to the recipient, the helpers are allocated different priorities to contend the right of cooperative transmission. Helpers with the same priority can use any of the existing collision resolution schemes, such as the $k$-round elimination contention $(k$-EC) scheme [15], to select a unique helper that is the best possible. In addition, the packet piggyback mechanism is used to improve multiple access performance. In other words, after the high data rate helper relays the data packet of the sender, it can transmit its own data packet to its recipient without channel reservation.

The contributions of this paper are as follows:

- Specifying a cooperative MAC protocol that integrates helper selection, packet piggyback and medium access for the application of cooperative communication technique to the MAC layer.

- Designing a helper selection scheme including a priority differentiation phase and contention resolution phase to promptly select a unique optimal helper from all potential helpers with high probability for further exploiting the cooperation gain. Any existing collision resolution scheme can be used in the contention resolution phase.

- Providing a packet piggyback mechanism to allow a high data rate helper to piggyback its own data packets to its recipient without reservation. This significantly decreases the reservation overhead and effectively improves cooperation efficiency and channel utilization.

The rest of the paper is organized as follows. In Section 2, related work is introduced. The network model is given in Section 3, while the proposed protocol is described in Section 4. Simulation results are presented in Section 5, followed by our conclusions in Section 6 .

\section{Related work}

The key problem of cooperative MAC protocols is how to select the best helper from multiple potential candidates, i.e., identifying the condition that the best helper must satisfy, the criteria for choosing a helper, and when to cooperate. Considering the cooperative participation manner of a helper, helper selection methods can be divided into three categories: the sender-specified method, the recipient-specified method, and the helper contention method.

Typical cooperative MAC protocols for a senderspecified helper include the CD-MAC [1], CoopMAC [8], rDCF [9], adaptive distributed cooperative MAC (ADC-MAC) [16], relay-contention-free cooperative MAC (RCF-CMAC) [17], the PTCoopMAC protocol [18], cooperative access with relay data (CARD) [19], and busy tone-based cooperative MAC [20] protocols. In the CD-MAC protocol [1], by eavesdropping on data packet transmissions of neighboring nodes, the sender can determine the SNR between itself and potential helpers. It then selects the neighboring node with the highest SNR as its helper and establishes a cooperation table. The sender binds with the helper until the associated cooperative transmission fails or the sender finds that there is a helper with better link quality. The drawbacks of the protocol are the following. First, the cooperation table requires large storage overhead. Second, information of the helpers cannot be updated timely. Finally, the data rate from the helper to the recipient cannot be guaranteed. Therefore, this protocol cannot achieve optimal cooperation gain. In the ADC-MAC protocol [16], each node establishes a cooperation table, uses the shortest path algorithm to select the helper between itself and its recipient, and designates the helper in a request-to-send (RTS) packet. If the received SNRs of the packets transmitted by the helper to the sender and recipient are higher than a given threshold, the helper sends a helper-clear-to-send (HCTS) packet to indicate its willingness to cooperate. The sender determines whether or not the helper can cooperate in transmission based on the instantaneous received SNRs from the sender to the helper and from the recipient to the helper recorded in the HCTS packet. If the helper is able to cooperate, the sender adopts the cooperation method to transmit the data packet. Otherwise, the sender transmits the data packet to its recipient directly. However, the protocol consumes additional communication overhead because each node periodically broadcasts heartbeat packets, and does not maximize cooperation performance improvement because the only helper nominated by the sender may decrease cooperation performance in actual cooperative transmission. In the RCF-CMAC protocol [17], a sender preselects two optimal helper candidates using its local relay information table and sets different priorities for them in a cooperative RTS (CRTS) packet based on the relay efficiency reflecting the level of its saved time. By eavesdropping on the handshakes between the sender and recipient, the two helper candidates can acquire instantaneous transmission rate information between the sender, recipient, and themselves. Then, according to their priorities and associated instantaneous transmission rate information, they orderly declare themselves available as the final relay. Thus, the proposed protocol can rapidly select the optimal relay under current channel quality without contention collisions to cooperatively transmit data packets from all the potential relays. Consequently, cooperative multiple access performance is improved. To resolve the performance degradation problem caused by the out-of-date information due to node mobility, the PTCoopMAC protocol [18] extends the CoopMAC protocol [8] with perceptron training to 
distinguish the helpers with low mobility from the helpers with high mobility by collecting history data on the signal strength of overheard packets. It can select the optimal helper depending on not only current overheard packets but also history statistics of the helper nodes.

One of the typical cooperative MAC protocols for a recipient-specified helper is the OXIDE protocol [21]. In this protocol, each node maintains a relay table, which records a potential helper's ID, the time of the last frame transmission overheard from the helper, the signal SNR from the node to the helper, and the number of cooperative transmission failures of the helper. When this last number exceeds a predefined threshold, the corresponding entry is removed from the relay table. If the sender fails to transmit the data packet to the recipient directly, the recipient chooses the node with the maximum mutual information of cooperative transmission from the relay table as the helper and sends a claim for cooperation packet to inform the sender of the transmission failure and notify the helper to proceed with cooperative transmission. The protocol guarantees only that the link quality from the helper to the recipient is optimal, but cannot ensure the link quality from the sender to the helper. As a result, the helper may not receive the data packet correctly from the sender, thus leading to cooperative transmission failure. To solve this problem, the cooperative relaying MAC (CoRe-MAC) protocol adopts the combination of a recipient-specified method and a helper contention method [22]. In the CoReMAC, the destination node estimates the received SNR of the RTS packet to determine whether or not to start a cooperative communication with a cooperative CTS (CCTS) packet. In the CCTS packet, the destination node specifies a prioritized relay set according to the early communication process. If the direct packet transmission fails, during the relay selection phase, all the prioritized relays that successfully received the data packet reply the busy tone in sequence, and then the destination node chooses the best relay with the maximum received SNR by estimating the received SNR of their busy tones. If there is no prioritized relay set, a new relay should be chosen. All relay candidates reply busy tone simultaneously and then the source node estimates the relay contention duration by the non-adaptive neighbor estimator. Each candidate sends an apply-forrelay (AFR) packet in a randomly chosen slot during candidate contention step. Then, the destination node chooses the candidate from which it received the AFR packet with the highest received SNR as relay for current cooperation attempt and selects all the candidates from which it received AFRs as prioritized relays for future communication attempts with associated source node.

Both the sender-specified helper and recipient-specified helper methods need to establish and maintain a relay table, resulting in possible outdated cooperation information and higher overhead. Recently, more and more cooperative MAC protocols make use of the helper selection method, that is, the helper contention method [4,13,23-25]. In the IrcMAC protocol [13], helpers are allocated one of eight different priorities. Helpers with different priorities send cooperation information at different times. Thus, the best helper can be determined based on its transmission time at the end of the cooperative contention phase. However, lower priority helpers also need to transmit their cooperation information, resulting in channel resource wastage. Moreover, multiple helpers may be selected to relay the data packet at the same time, which requires strict time synchronization and higher power consumption. The CoopMAC-aggregation (CoopMACA) protocol [24] uses a packet aggregation mechanism to improve throughput performance. Its helper selection procedure includes a classification round, priority round, and contention round. The classification round distinguishes the helpers with a packet to send and those without a packet to send. Helpers with a data packet to send take precedence in entering the priority round. In this round, helpers with the highest data rates to the sender and recipient are selected, and then in the contention round, a unique best helper is determined from all the highest priority helpers. Finally, the selected helper with a packet to send adopts the packet aggregation mechanism to carry out cooperative transmission, i.e., the selected helper aggregates its data packet with the sender's data packet and sends the aggregated data packet to the recipient. In the case that multiple helpers are selected, cooperative transmission cannot be used, and the sender transmits the data packet directly to the recipient. The packet aggregation mechanism cannot be used either, which results in channel wastage due to cooperation handshakes and higher cooperation overhead. In addition, the protocol first selects all nodes with a packet to send as helper candidates and then considers cooperative performance improvement. Therefore, cooperative multiple access performance is not always optimal and is likely to be worse. In the CoopGeo protocol [25], each node contends the cooperation right according to geographic information, thus obviating the need for cooperative control packet handshakes. The helper in the best cooperative location between the sender and its recipient has a counter with the smallest value and can relay the data packet earlier. This protocol, therefore, avoids control packet collisions and requires less overhead. However, it is possible that the counter values of multiple helpers could be almost the same, leading to cooperative transmission failure.

\section{Network model}

In a multihop wireless network, all nodes are randomly distributed in a given area and share one wireless 
symmetrical channel. Each node exchanges control packets and data packets on the channel using a half-duplex transceiver with fixed transmission power. If there is no packet to send, a node continually senses the channel.

The PHY layer of each node provides multirate transmission capability. For simplicity, we consider the IEEE 802.11b PHY layer [26] in this paper, which can support multirate transmission of 1, 2, 5.5, and 11 Mbps. Each node sends RTS, CTS, and acknowledgment (ACK) packets at the basic transmission rate of $1 \mathrm{Mbps}$ and data packets at the maximum transmission rate to its recipient. Each node can calculate the corresponding data rate satisfying a certain BER between it and a neighboring node by means of the reception of the neighboring node's packets [8]. If multiple helpers simultaneously start transmitting the same data packet to a recipient, the recipient can correctly receive the data packet.

\section{RCD-MAC protocol}

The RCD-MAC protocol uses a helper with a high data rate to assist a sender with a low data rate to transmit data packets, thereby realizing cooperation gain. The protocol comprises three phases, namely, the reservation phase, helper selection phase, and data packet transmission phase. The helper selection phase is further divided into a priority differentiation phase and a contention resolution phase involving the same priority helpers, which can promptly select a unique optimal helper to improve cooperation efficiency. The proposed protocol also adopts a packet piggyback mechanism, i.e., a high data rate helper with a packet to send can piggyback its reservation information on the data packet relayed on behalf of the sender, which means that its data packet is sent to its recipient immediately after the end of the cooperative data packet transmission without further reservation handshakes. Here, the recipients of the sender and helper can be different. Thus, the mechanism greatly decreases reservation overhead, provides rapid channel access for the helpers, and further improves multiple access performance.

\subsection{Reservation phase}

When a sender $S$ senses that the channel is idle, it sends an RTS packet to its recipient $D$ at the basic data rate of 1 Mbps according to 802.11 DCF [26]. On receiving the RTS packet correctly, the recipient replies to its sender with a CTS packet. From the RTS/CTS handshakes, both the sender and recipient know the highest data rate $R_{\mathrm{SD}}$ supported between each other. Moreover, any neighboring node in the network who successfully receives and decodes both the RTS and CTS packets can also estimate the highest data rate between the sender and itself (i.e., $R_{\mathrm{SH}}$ ), and the highest data rate between itself and the recipient (i.e., $R_{\mathrm{HD}}$ ). If $R_{\mathrm{SD}}$ is in the high data rate group, i.e., 11 or $5.5 \mathrm{Mbps}$, the sender transmits the data packet to the recipient directly, as shown in Figure 1. Otherwise, it adopts cooperative transmission to send the data packet and thus initiates the helper selection phase.

\subsection{Helper selection phase}

The helper selection phase consists of a priority differentiation phase and a contention resolution phase involving the same priority helpers.

\subsubsection{Priority differentiation phase}

If the helper does not receive a data packet in the interval SIFS $+\tau$ after it has received the CTS packet, this indicates that the sender is unable to support high data rate transmission to its recipient. Therefore, the helper needs to initiate cooperative transmission and starts the priority differentiation phase. The reserved $\tau$ allows the helper to decide whether or not it needs to cooperate and to avoid collisions between cooperative contention transmission and direct data packet transmission.

In the priority differentiation phase shown in Figure 2, the helpers are divided into 12 different priority levels based on the following two factors for cooperative participation: the values of $R_{\mathrm{SH}}$ and $R_{\mathrm{HD}}$, and whether or not the helper has a packet to send. The helper with the highest priority can choose an earlier minislot to send its busy tone. The first, second, third, and fourth level priority helpers are those with a packet to send and a higher $R_{\mathrm{SH}}$ and $R_{\mathrm{HD}}$. The fifth, sixth, seventh, and eighth level priority helpers are those without a packet to send, but with a higher $R_{\mathrm{SH}}$ and $R_{\mathrm{HD}}$. The ninth and tenth level priority helpers are those with a packet to send, but with a lower $R_{\mathrm{SH}}$ and higher $R_{\mathrm{HD}}$. The 11th and 12th level priority helpers are those with a lower $R_{\mathrm{SH}}$ and higher $R_{\mathrm{HD}}$, or a higher $R_{\mathrm{SH}}$ and lower $R_{\mathrm{HD}}$. As shown in Figure 2, if both $R_{\mathrm{SH}}$ and $R_{\mathrm{HD}}$ are $11 \mathrm{Mbps}$, and the helper has a packet to send, it is allocated the first priority. The probabilities of $R_{\mathrm{SH}}$ and $R_{\mathrm{HD}}$ for the first ten priorities are unique. However, the last two priorities have two possible cases because both cases have the same cooperation efficiency. For example, the 11th priority includes the cases, where $R_{\mathrm{SH}}=2 \mathrm{Mbps}$ and $R_{\mathrm{HD}}=11 \mathrm{Mbps}$,

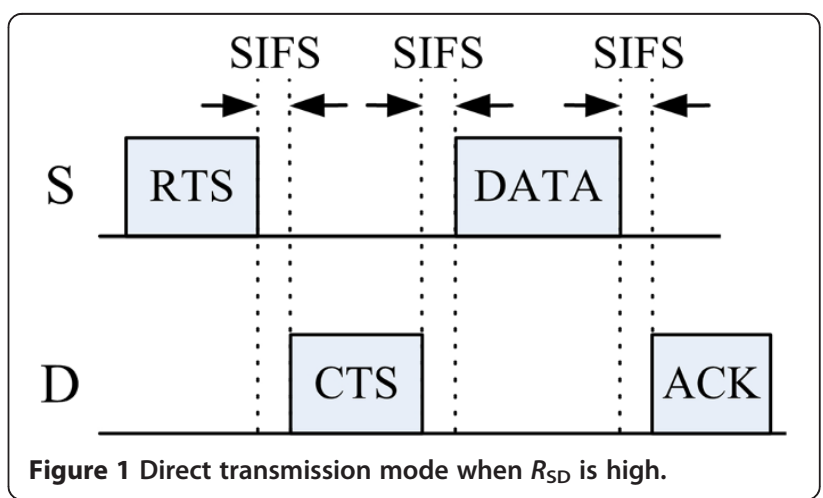




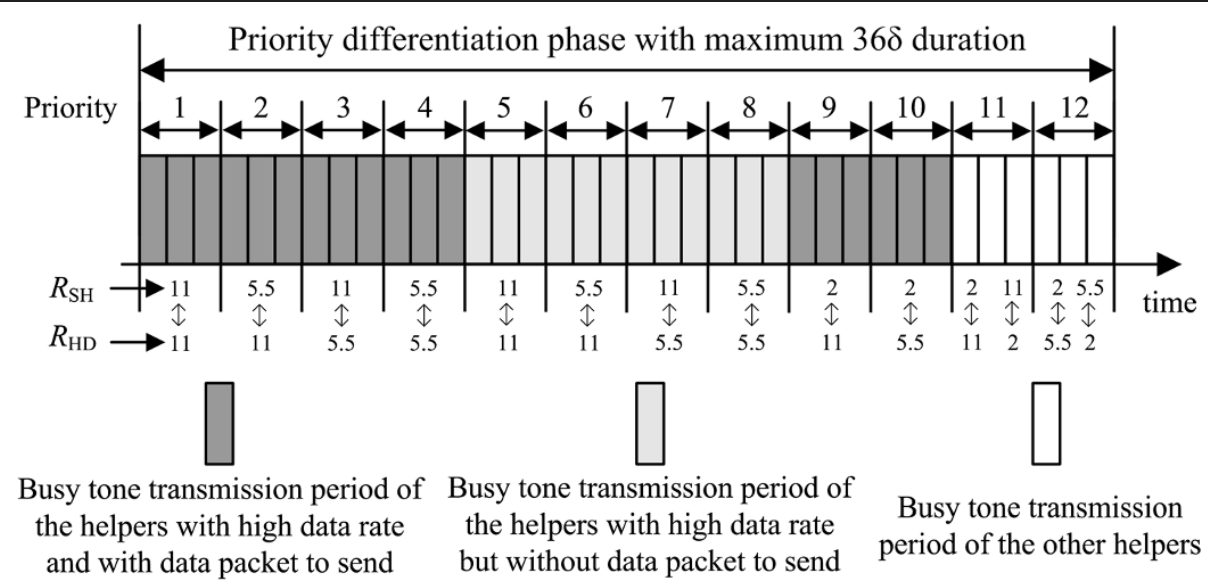

Figure 2 Priority differentiation phase.

and $R_{\mathrm{SH}}=11 \mathrm{Mbps}$ and $R_{\mathrm{HD}}=2 \mathrm{Mbps}$. Each priority has three minislots with interval $\delta$.

By eavesdropping on the RTS and CTS packets, each helper estimates the highest data rates $R_{\mathrm{SH}}$ and $R_{\mathrm{HD}}$ that it can support and randomly chooses one of three minislots for the corresponding priority to send a busy tone with interval $\delta$. Every helper must sense the busy tone transmission when it is free during the procedure. If the helper senses a busy tone transmission before its predefined busy tone transmission, it does not send its busy tone and withdraws from cooperation contention, owing to the already-successful helper with the same or higher priority. The priority differentiation phase ends when a helper sends its busy tone, and the contention resolution phase starts immediately in the next minislot. If one of the first priority helpers selects the first minislot of the corresponding three minislots to send its busy tone, the priority differentiation phase ends after the $\delta$ interval. Thus, the priority differentiation phase has a minimum interval $\delta$ and a maximum interval $36 \delta$.

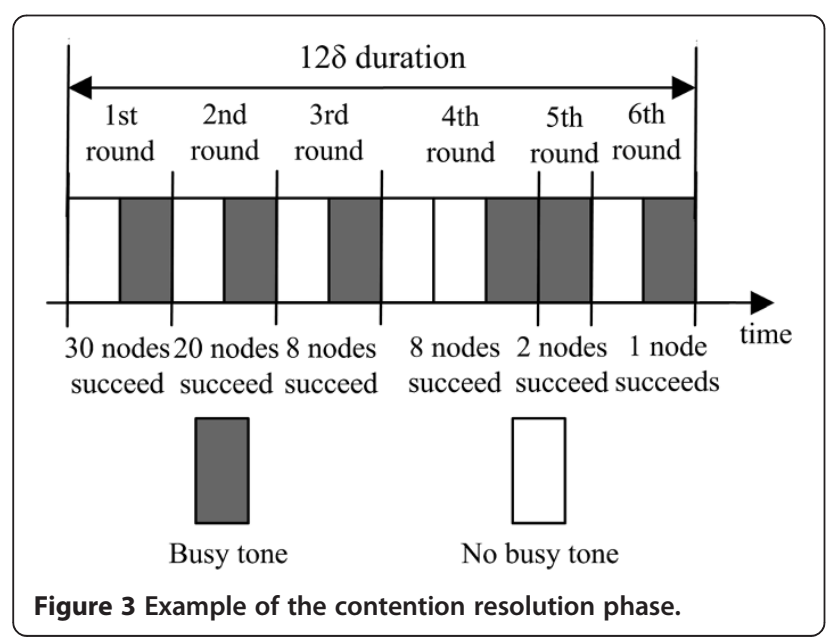

For instance, helper $R_{1}$ with a packet to send estimates that $R_{\mathrm{SH}}$ is $11 \mathrm{Mbps}$ and $R_{\mathrm{HD}}$ is $5.5 \mathrm{Mbps}$, and does not sense any busy tone before its upcoming busy tone transmission. Therefore, it sends its busy tone in a minislot randomly selected from the three minislots for the third priority. Other helpers sense the busy tone and cancel their own impending busy tone transmissions. Thus, helper $R_{1}$ gets the opportunity to enter the contention resolution phase to contend the cooperation right.

\subsubsection{Contention resolution phase}

It is possible that multiple helpers with the same priority choose the same minislot to simultaneously send busy tones. To avoid possible collisions caused by their concurrent cooperative packet transmissions and guarantee application of the packet piggyback mechanism, the contention resolution phase among the same priority helpers is employed. Any collision resolution scheme can be used for this. For the sake of simplicity and efficient channel access, this paper adopts the $k$-EC scheme [15], which consists of $k$ rounds of collision elimination procedures with at most $m$ contention minislots in each round.

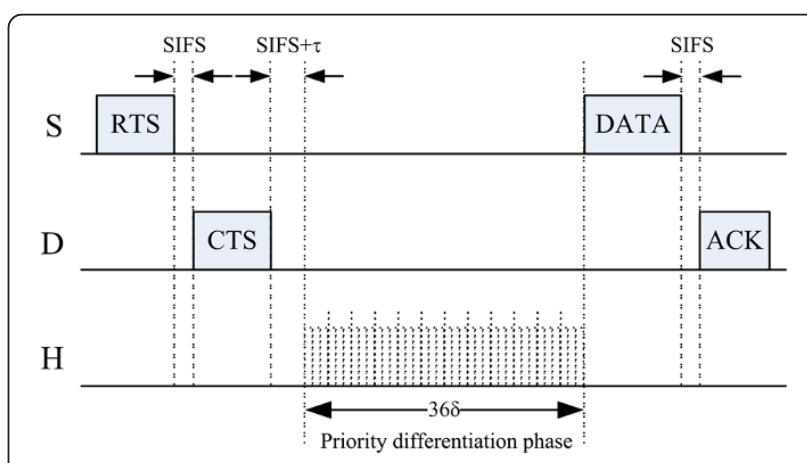

Figure 4 The case of no helper for cooperative transmission. 


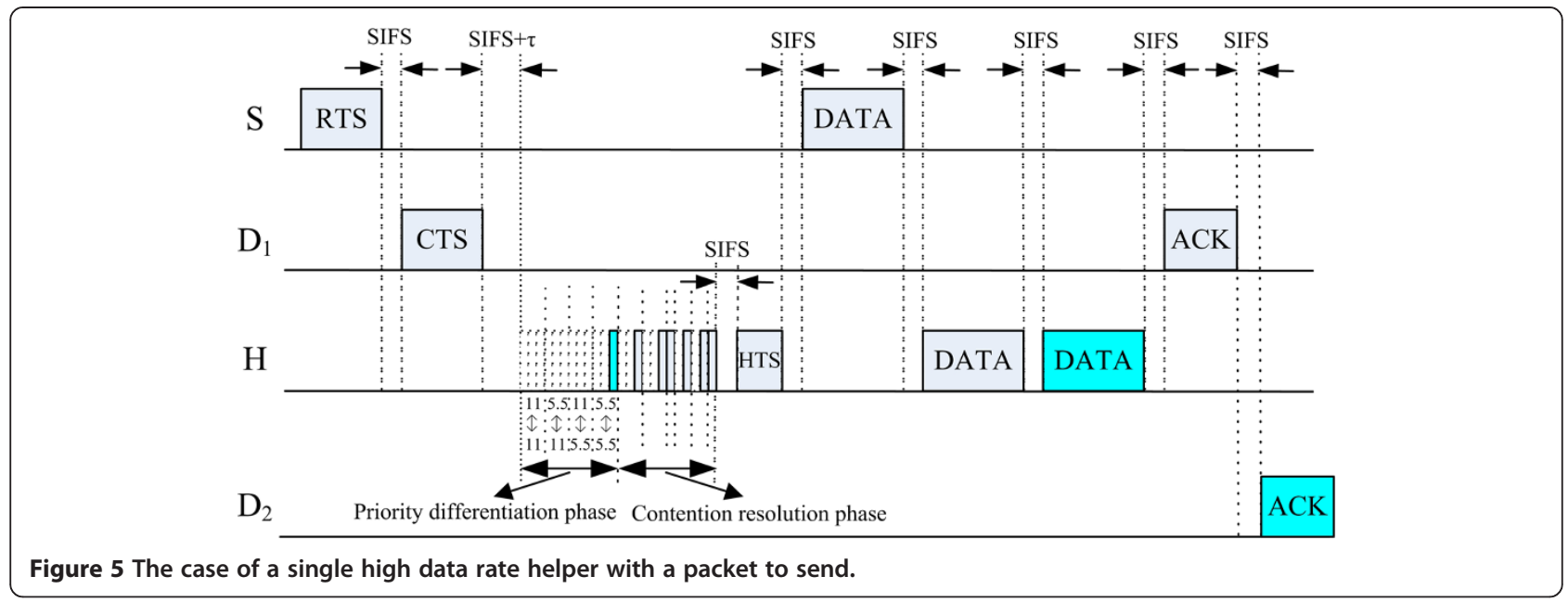

All successful helpers in the priority differentiation phase randomly choose one of the $m$ minislots in the first round to send a busy tone. If a helper senses a busy tone before its busy tone transmission, it withdraws from contention and cancels its scheduled busy tone transmission. Helpers that succeed in sending a busy tone in the earliest minislot of the round immediately repeat the procedure in the next and subsequent rounds until the end of the $k$-th round. Each contention resolution round ends as soon as one helper sends the busy tone, and the next round of contention starts immediately among the successful helpers. In the $k$-EC scheme [15], the optimal values of $m$ and $k$ are 3 and 7, respectively, allowing a unique helper to be selected with probability of at least $97.7 \%$ in the case of 100 contending nodes and network throughput to be maximized for most cases in real networks. Because the proposed protocol carries out one round of the collision resolution process in the priority differentiation phase, the optimal $k$ is 6 for the contention resolution phase among helpers with the same priority. For each minislot with interval $\delta$, the contention resolution phase among the same priority helpers has a minimum length of $6 \delta$ and maximum length of $18 \delta$. Figure 3 shows an example of the contention resolution phase.

\subsection{Data packet transmission phase}

Based on the different helper selection results, the data packet transmission phase is divided into four cases as follows:

(1) As shown in Figure 4, if no helper is found in the network after the helper selection phase terminates,

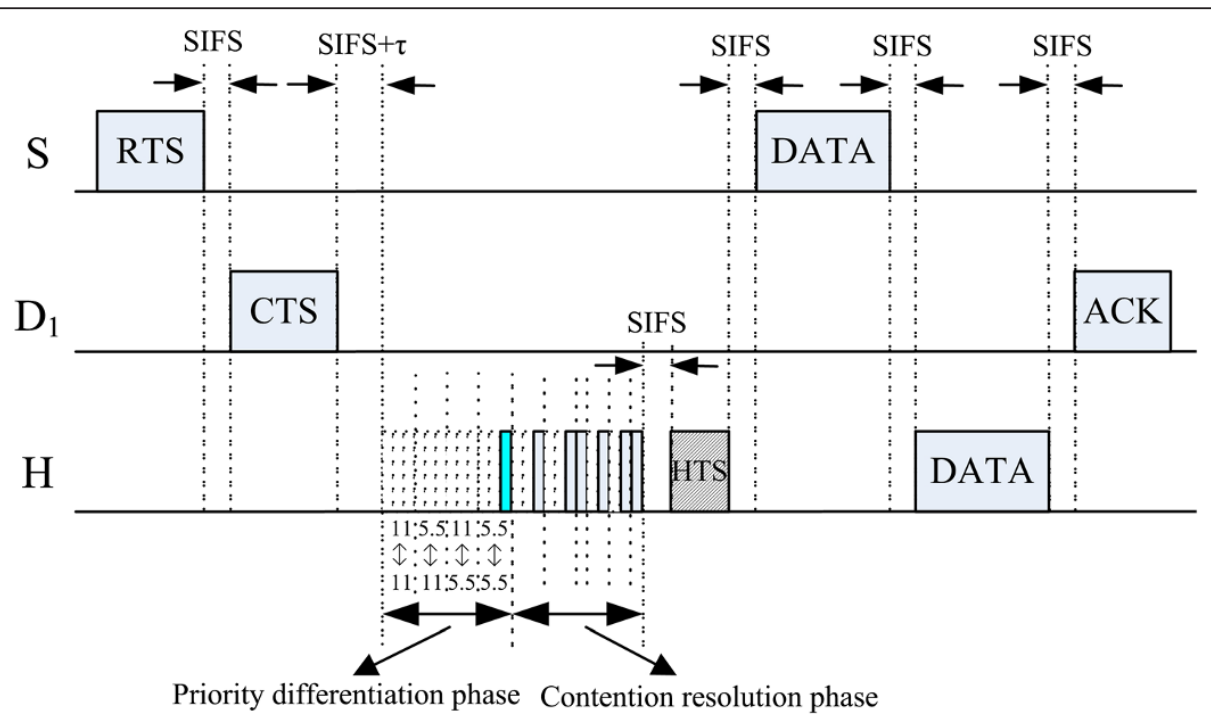

Figure 6 The case of multiple high data rate helpers with packets to send. 


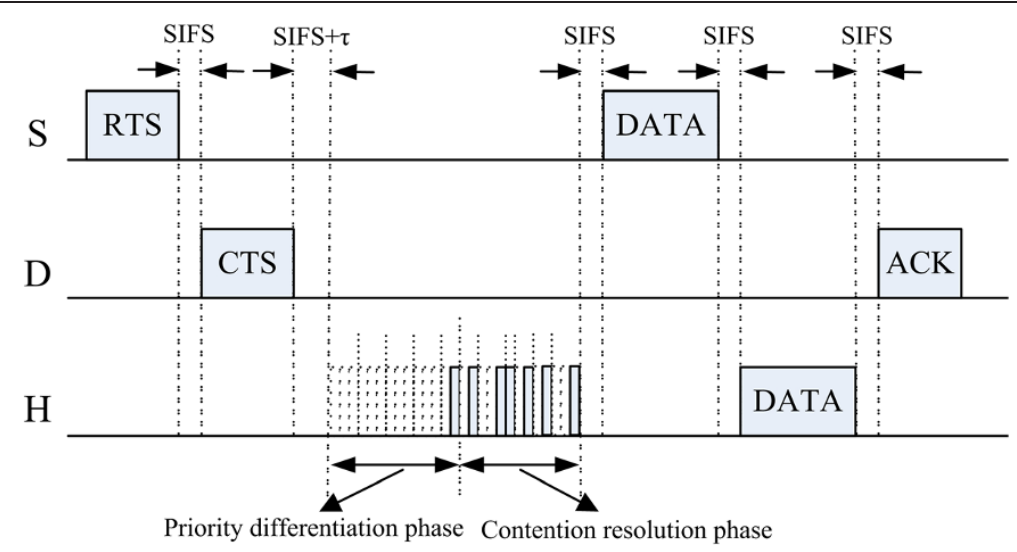

Figure 7 The case of high data rate helper(s) without packets to send.

the sender transmits its data packet at a data rate $R_{\mathrm{SD}}$ directly to its recipient.

(2) If the successful helpers support a high data rate and have data packets to send (i.e., a helper with priority $1,2,3,4,9$, or 10 ), the packet piggyback mechanism can be used. However, it is vital that only a single best helper wins during the helper selection phase. As shown in Figure 5, to achieve this, every successful helper needs to send a helpto-send (HTS) packet to the sender at a data rate $R_{\mathrm{SH}}$ at the end of the helper selection phase. If the sender successfully receives and decodes the HTS packet, this indicates that only one helper has won during the helper selection phase, and the packet piggyback mechanism can be used. The information of the packet piggyback mechanism and the total transmission time for the entire data packet transmission are designated in the data packet transmitted by the sender at a data rate $R_{\mathrm{SH}}$. After the helper receives the data packet from the sender, it relays the data packet to the recipient of the sender at a data rate $R_{\mathrm{HD}}$ and then transmits its own data packet to its recipient. The recipients of the sender and helper reply in turn with an ACK packet to their respective senders.

(3) In case (2), as shown in Figure 6, if the sender is unable to decode the HTS packet, this indicates that multiple helpers have succeeded during the helper selection phase and have sent HTS packets simultaneously resulting in collisions at the sender. In this case, the packet piggyback mechanism cannot be used. The sender therefore, transmits the data packet to all successful helpers at a data rate $R_{\mathrm{SH}}$, and the helpers relay it to the recipient at a data rate $R_{\mathrm{HD}}$. The recipient replies to the sender with an ACK packet after it has successfully received the data packet.
(4) If the successful helpers support a high data rate but do not have data packets to send (i.e., a helper with priority $5,6,7,8,11$, or 12 ), the packet piggyback mechanism cannot be used. The sender transmits its data packet to the helpers at a data rate $R_{\mathrm{SH}}$ immediately after the end of the helper selection phase, and the helpers subsequently relay the data packet to the recipient at a data rate $R_{\mathrm{HD}}$. The recipient replies with an ACK packet to the sender after it has successfully received the data packet. The transmission process is shown in Figure 7.

\section{Performance evaluation}

In this study, we used the $\mathrm{C}$ programming language to simulate the performance of the RCD-MAC protocol and compared it with the performance of the IrcMAC

Table 1 Simulation parameters

\begin{tabular}{lc}
\hline Parameter & Value \\
\hline MAC header & 272 bits \\
PHY header & 192 bits \\
Data rate for MAC and PHY headers, and RTS, & $1 \mathrm{Mbps}$ \\
CTS, HTS, and ACK packets & \\
RTS & 160 bits \\
CTS/ACK & 112 bits \\
HTS & 112 bits \\
SIFS/T & $10 \mu \mathrm{s}$ \\
DIFS & $50 \mu \mathrm{s}$ \\
$\delta$ & $1 \mu \mathrm{s}$ \\
Slot time & $20 \mu \mathrm{s}$ \\
Maximum number of retransmissions & 6 \\
Packet lifetime & $0.512 \mathrm{~s}$ \\
\hline
\end{tabular}




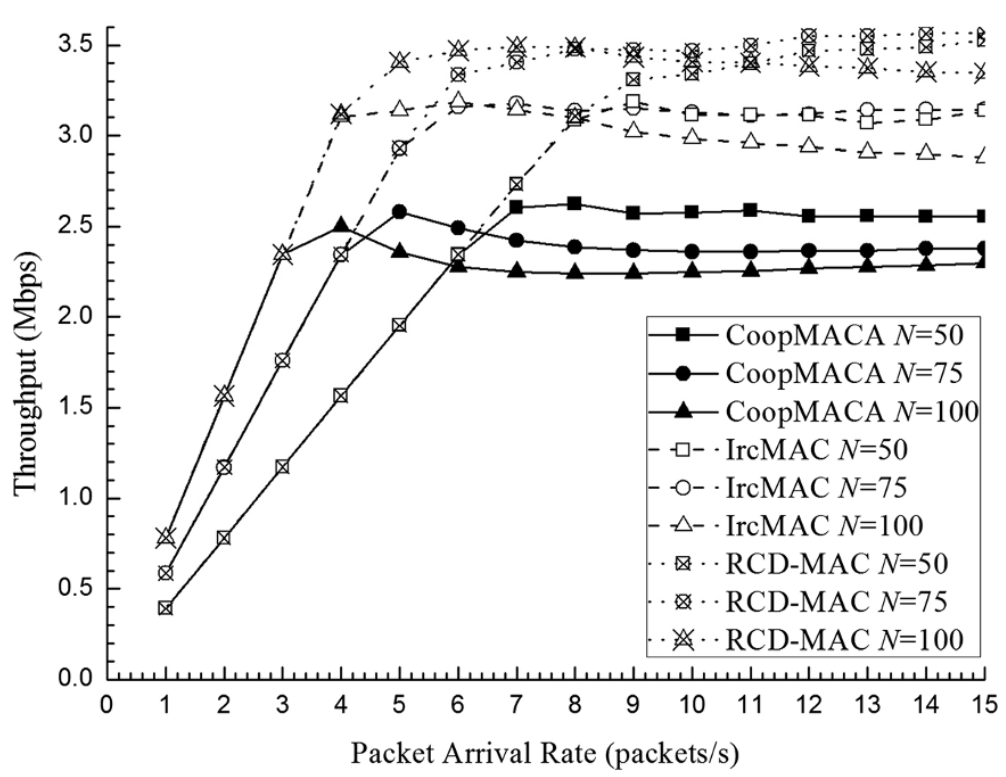

Figure 8 Throughput with a varying number of nodes.

and CoopMACA protocols. In the simulation, we used throughput, saturation throughput, and delay as performance metrics to evaluate multiple access performance. Herein, throughput is defined as all the successfully transmitted data packets in bits per second, saturation throughput is defined as the successfully transmitted data packets in bits per second, given that each node always has a packet to transmit, and delay is defined as the mean lifetime of a data packet from its generation to its successful reception by the recipient.

\subsection{Simulation environment}

In the simulation, two typical wireless networks, namely a WLAN and an ad hoc network, with $N$ nodes are considered. All nodes are randomly distributed in a circular area with a radius of $100 \mathrm{~m}$ in the network. In the WLAN, an access point (AP) is located at the center of the circle, and all the sender's recipients are the AP, whereas in the ad hoc network, the recipient of a sender is randomly selected from its neighboring nodes. The networks can support data rates of $11,5.5,2$, and 1

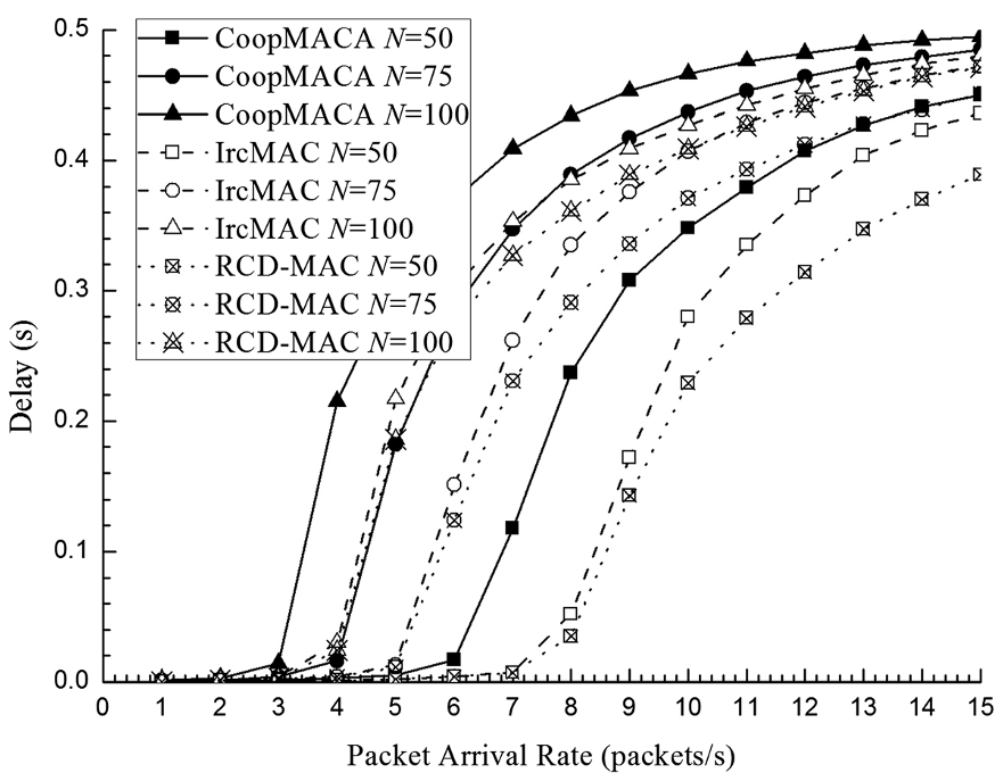

Figure 9 Packet delay with a varying number of nodes. 


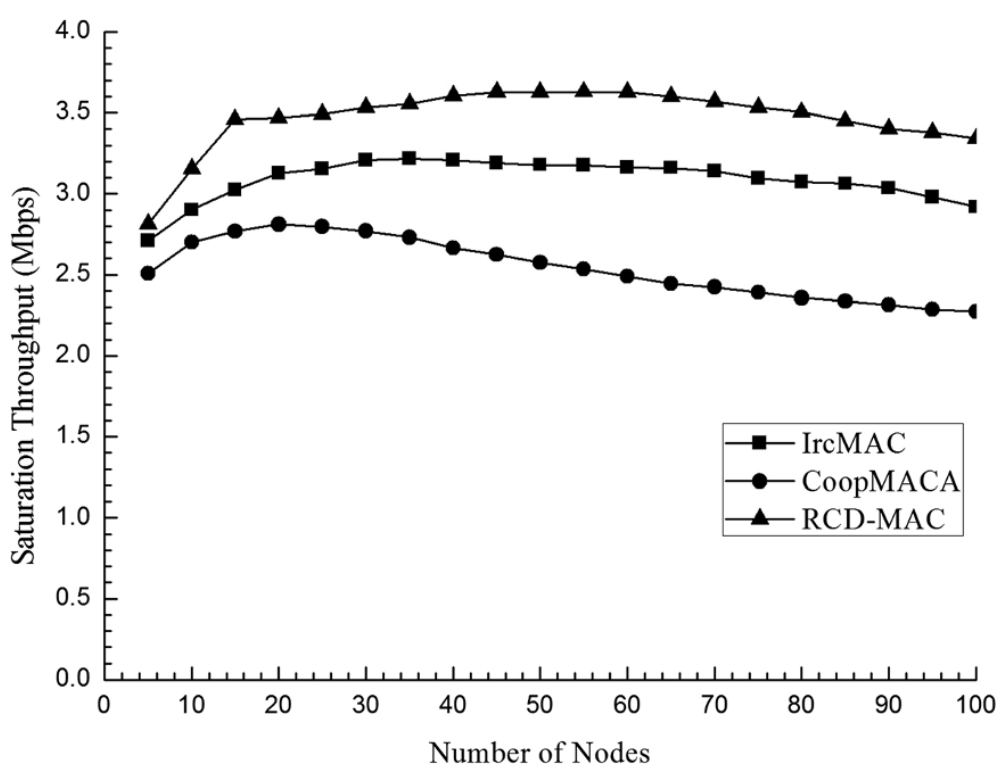

Figure 10 Saturation throughput with a varying number of nodes.

Mbps according to the IEEE 802.11b standard. Packets are transmitted at different rates, depending on the distance between the sender and the recipient. Under the condition that the path loss exponent is 3 and the BER is lower than $10^{-5}$, the maximum transmission distances at data rates $11,5.5,2$, and 1 Mbps are 48.2, 67.1, 74.7, and $100 \mathrm{~m}$, respectively [8]. Each node generates data packets with a fixed length according to a Poisson distribution with packet arrival rate $\lambda_{0}$. By default, the length of a data packet $L_{\mathrm{PKT}}$ is $1 \mathrm{kB}$, and $N$ is 100 . The simulation parameters are listed in Table 1 . Here, $\delta$ is set to
$1 \mu$ s according to the IrcMAC protocol [13]. To enhance accuracy, we reported the average simulation results over 50 random network topologies as our final results. To fully reflect protocol performance, we considered transmission failures caused only by packet collisions, and not those due to channel errors.

\subsection{Impact of $N$ on network performance in a WLAN environment}

Figures 8 and 9 show the performance comparison of the RCD-MAC, CoopMACA, and IrcMAC protocols when

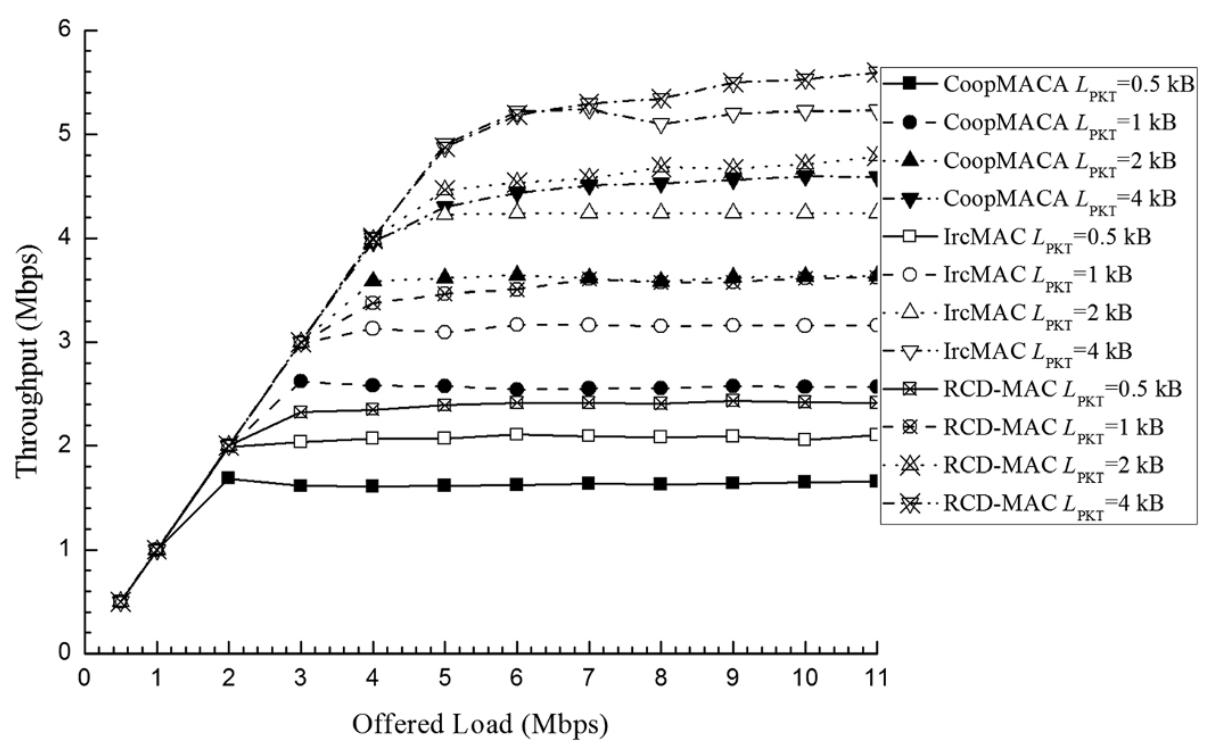

Figure 11 Throughput with varying packet lengths. 


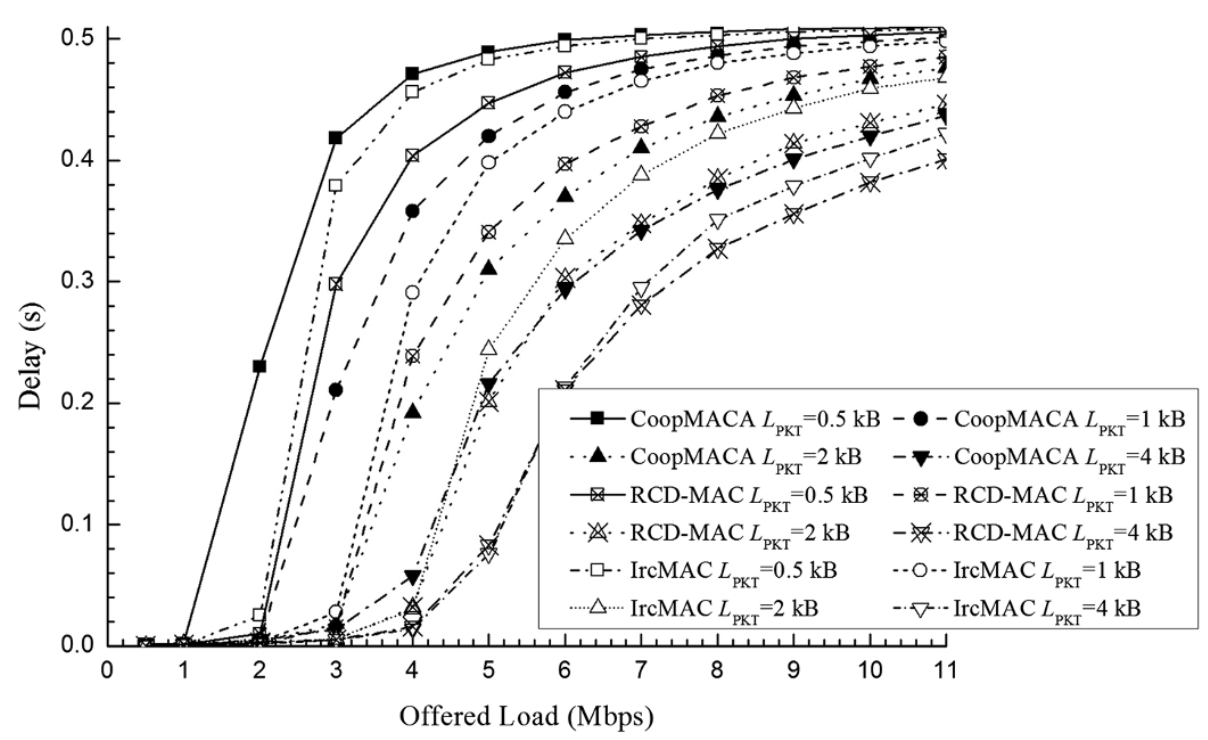

Figure 12 Packet delay with varying packet lengths.

varying the number of nodes $N$ and the packet arrival rate in a WLAN. Figure 10 shows the impact of $N$ on the saturation throughput with 10 -Mbps offered load. These figures show that when the packet arrival rate is small, an increase in $N$ causes the throughput and saturation throughput of all protocols to increase and their delays to increase slightly. However, as the packet arrival rate increases, especially when the network approaches saturation, an increase in $N$ causes the throughput and saturation throughput of all protocols to decrease slightly and their delays to increase drastically. This is because at a low offered load, with an increase in $N$, more data packets can be transmitted successfully in time. However, as the network becomes saturated, the offered load increases with an increase in $N$, which leads to more packet collisions, and thus higher service time for each data packet and lower throughput. The proposed protocol keeps the throughput stable because it adopts the packet piggyback mechanism and has lower cooperation overhead and higher cooperation gain, resulting in better compensation for the performance decrease due to packet collisions.

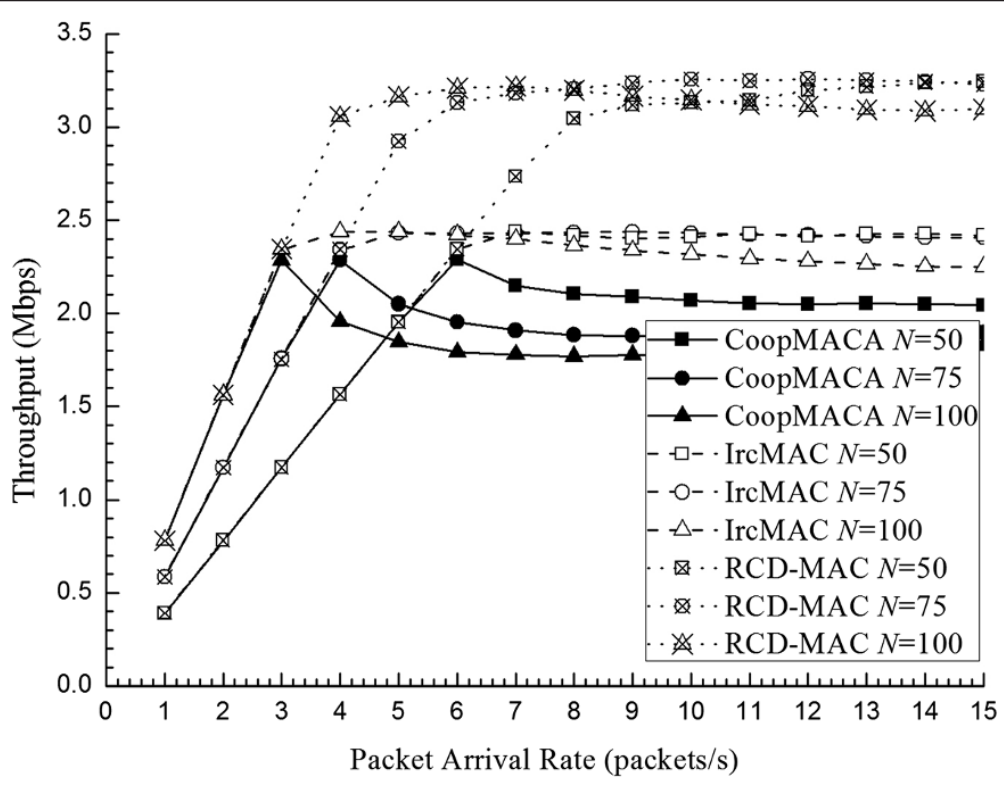

Figure 13 Throughput with a varying number of nodes. 


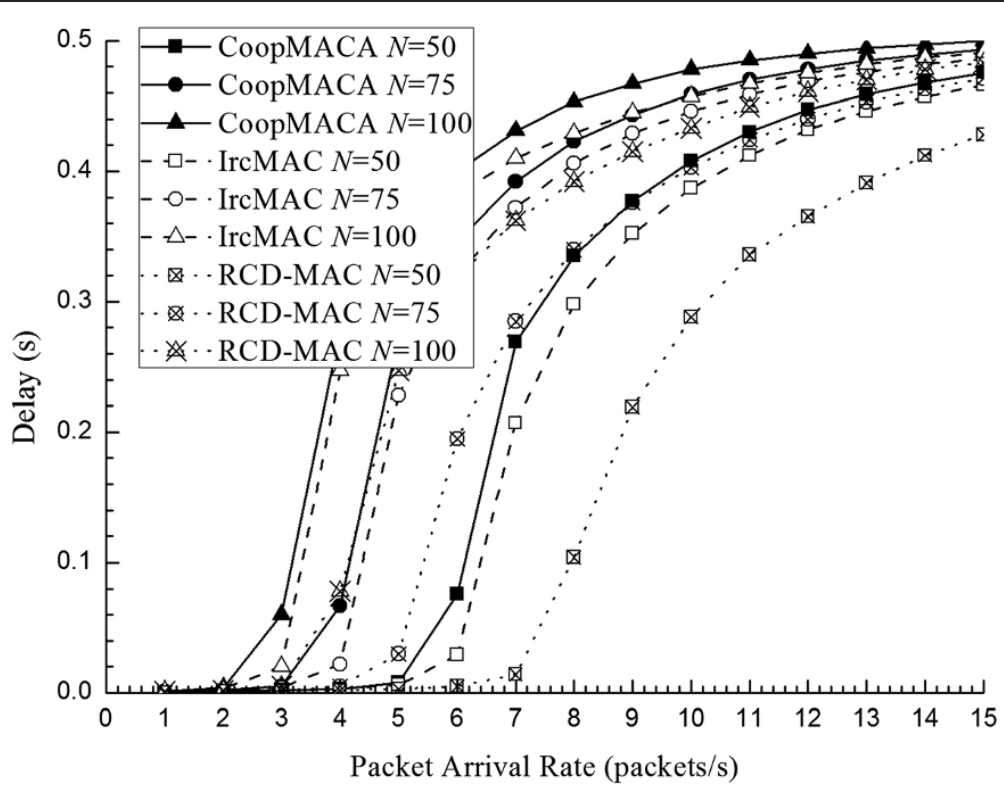

Figure 14 Packet delay with a varying number of nodes.

The figures also show that the throughput and saturation throughput of the RCD-MAC protocol are obviously higher than those of the CoopMACA and IrcMAC protocols. When $N$ is 100 , the maximum throughput of RCD-MAC is $39.6 \%$ and $9.6 \%$ higher than those of CoopMACA and IrcMAC, respectively, while the maximum saturation throughput is $29.1 \%$ and $12.7 \%$ higher than those of CoopMACA and IrcMAC, respectively. This is because RCD-MAC adopts a more efficient helper selection mechanism and the packet piggyback mechanism to improve transmission efficiency. In the proposed protocol, the priority differentiation phase can terminate early as long as one or more helpers with a higher priority participate in the contention. Therefore, RCD-MAC has the shortest helper selection phase with a maximum time of $54 \mu \mathrm{s}$, minimum time of $7 \mu \mathrm{s}$, and average time of $31 \mu \mathrm{s}$. The helper selection process of IrcMAC consumes a fixed time of $60 \mu \mathrm{s}$. In addition, it needs to initiate RTS/CTS handshakes for every data packet. As a result, it has greater overhead than RCD-MAC. CoopMACA can adopt the packet aggregation mechanism only when a single helper wins the cooperation transmission right. Otherwise,

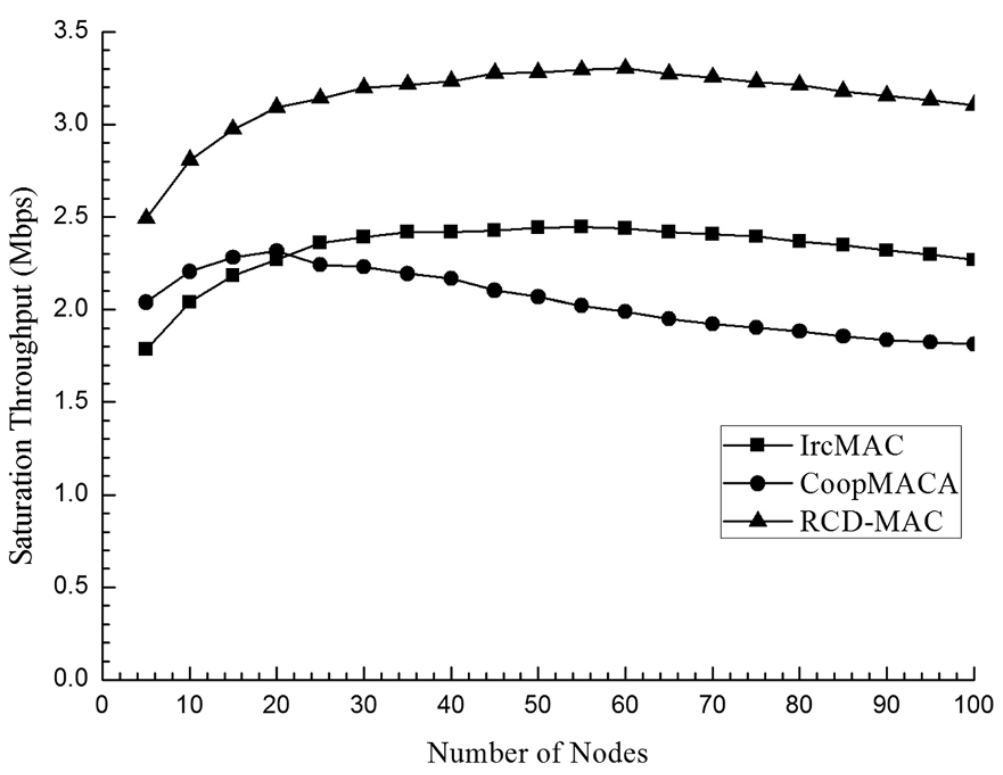

Figure 15 Saturation throughput with a varying number of nodes. 


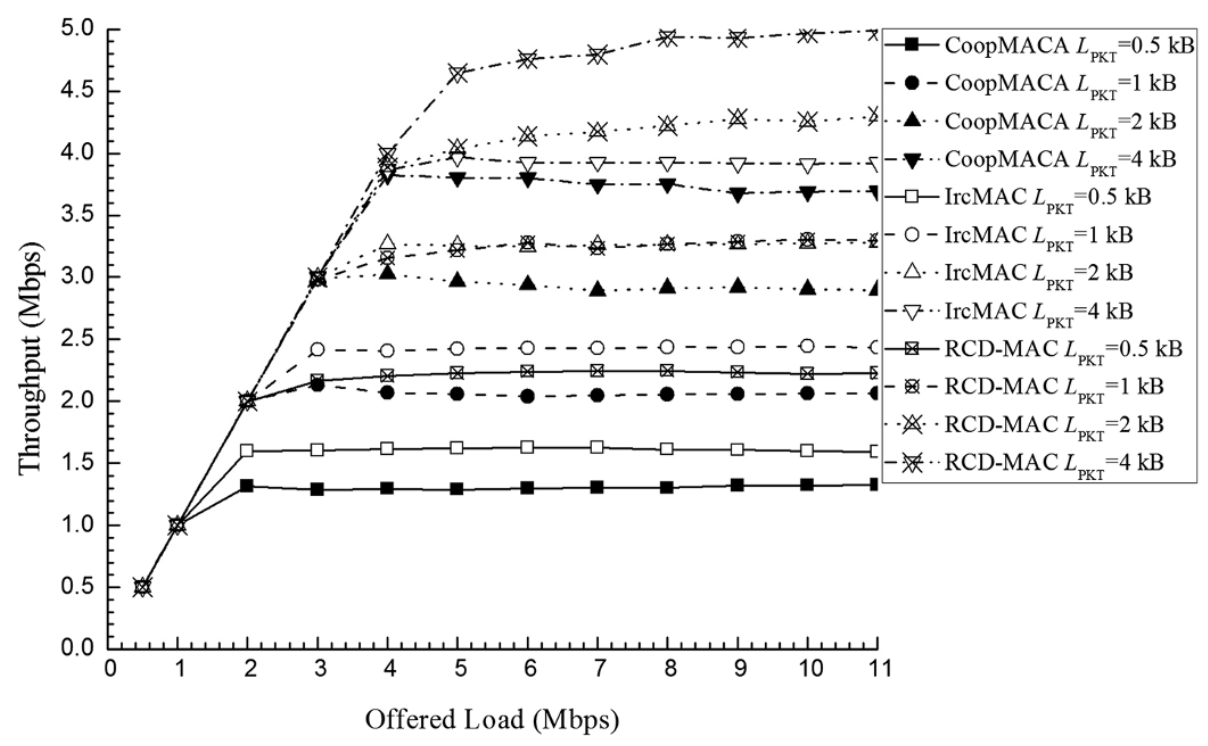

Figure 16 Throughput with varying packet lengths.

it uses direct transmission mode, which results in more cooperation overhead and does not improve the data rate from the sender to the recipient. It is most likely that multiple helpers will succeed during its three-round contention resolution procedure according to the $k$-EC scheme [15]. Meanwhile, the helper selection process takes an average time of $90 \mu \mathrm{s}$, which is slower than those of IrcMAC and RCD-MAC. Moreover, CoopMACA initially selects helpers with packets to send, rather than helpers with the best cooperation performance. Therefore, its performance is the worst. As mentioned before, RCD-MAC has the fastest helper selection process, which is most likely to select the best helper, and uses the packet piggyback mechanism to decrease reservation overhead, resulting in the highest throughput and lowest average packet delay of the three cooperative MAC protocols.

\subsection{Impact of $L_{\mathrm{PKT}}$ on network performance in a WLAN environment}

Figures 11 and 12 illustrate the impact of $L_{\mathrm{PKT}}$ on the performance of the RCD-MAC, CoopMACA, and IrcMAC protocols when varying the offered load in a WLAN. As

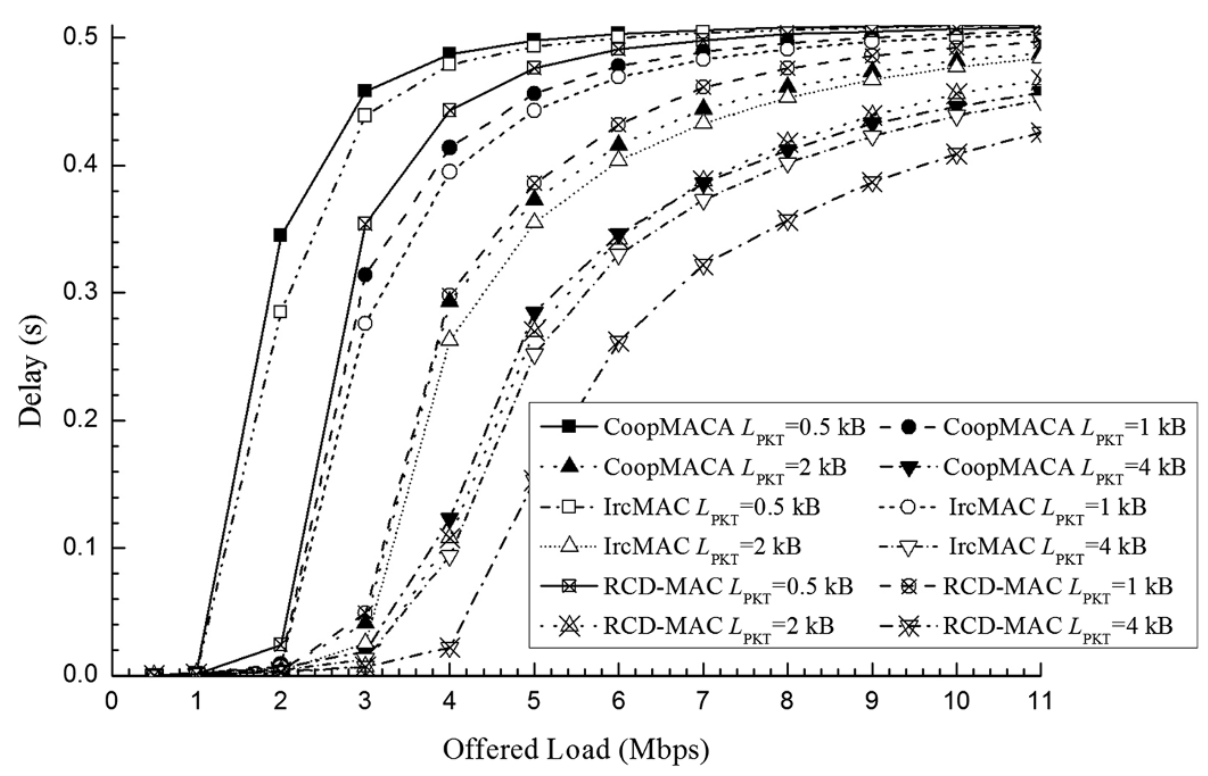

Figure 17 Packet delay with varying packet lengths. 
shown in these figures, an increase in $L_{\mathrm{PKT}}$ causes the throughput of all protocols to increase and their delays to decrease. This is because with an increase in $L_{\mathrm{PKT}}$, each node can send more data information bits with each successful channel reservation. Nevertheless, the performance of RCD-MAC is obviously better than that of the other protocols for different $L_{\mathrm{PKT}}$ values because it can select a unique best helper or the best helpers faster, while its packet piggyback mechanism effectively decreases reservation overhead. When $L_{\mathrm{PKT}}$ is $1 \mathrm{kB}$, RCD-MAC achieves a $40 \%$ and $14.3 \%$ throughput gain over CoopMACA and IrcMAC, respectively.

\subsection{Impact of $N$ on network performance in an ad hoc network environment}

Figures 13, 14, and 15 show the performance comparison of the RCD-MAC, CoopMACA, and IrcMAC protocols, respectively, when varying $N$ in an ad hoc network. Similar to the WLAN environment, the performance of RCD-MAC is superior to those of CoopMACA and IrcMAC. When $N$ is 100 , the maximum throughput of RCD-MAC is $40.8 \%$ and $31.9 \%$ higher than those of CoopMACA and IrcMAC, respectively, and its maximum saturation throughput is $42.7 \%$ and $35 \%$ higher than those of CoopMACA and IrcMAC, respectively. It is also obvious that the performance of IrcMAC and CoopMACA decreases markedly in ad hoc networks compared with that in WLANs, while RCDMAC maintains high performance. As mentioned before, in RCD-MAC, the data packets of the sender and its helper are transmitted separately, and their recipients can be different. Therefore, its packet piggyback mechanism can be applied similarly to both WLANs and ad hoc networks. However, the packet aggregation mechanism adopted by CoopMACA cannot be applied satisfactorily to ad hoc networks because it requires that the recipients of the data packets of the sender and the helper are the same, which leads to the inferior performance in ad hoc networks compared with that in WLANs. IrcMAC yields similar results because different communication node pairs in ad hoc networks greatly increase the probability of packet collisions. In addition, RCD-MAC has a smaller packet delay than CoopMACA and IrcMAC because it can access cooperative transmission more quickly, and its selected helper(s) can support higher data rates, thereby further decreasing the service time of data packets.

\subsection{Impact of $L_{\mathrm{PKT}}$ on network performance in an ad hoc network environment}

Figures 16 and 17 show the performance comparison of the RCD-MAC, CoopMACA, and IrcMAC protocols, respectively, for different $L_{\mathrm{PKT}}$ values in ad hoc networks. Similar to the results in a WLAN, an increase in
$L_{\mathrm{PKT}}$ causes the throughput of all three protocols to increase and their delays to decrease. It is obvious that RCD-MAC outperforms the other protocols. When $L_{\mathrm{PKT}}$ is $1 \mathrm{kB}, \mathrm{RCD}-\mathrm{MAC}$ achieves a $55 \%$ and $35.5 \%$ throughput gain over CoopMACA and IrcMAC, respectively. The above results verify that RCD-MAC can be suitably applied to both ad hoc networks and WLANs.

\section{Conclusions}

In this paper, we proposed a novel RCD-MAC protocol for multihop wireless networks. The proposed protocol adopts an efficient priority differentiation scheme and contention resolution scheme for rapidly selecting a unique optimal helper with the highest probability, thereby improving cooperation efficiency. Furthermore, it also uses a packet piggyback mechanism to reduce reservation overhead, expedite data packet transmission, and improve protocol performance. Simulation results on networks with 100 nodes show that compared with the CoopMACA and IrcMAC protocols, the proposed protocol achieves throughput improvement of 39.6\% and $9.6 \%$, respectively, in a WLAN environment, and $40.8 \%$ and $31.9 \%$, respectively, in an ad hoc network environment. In the future, we intend researching a new contention resolution scheme or joint priority differentiation and contention resolution scheme to further improve cooperation performance.

\section{Competing interests}

The authors declare that they have no competing interests.

\section{Acknowledgements}

We wish to thank Zheng Zheng for his comments and suggestions. We also express our thanks to the anonymous reviewers for their insightful comments to improve the quality and the presentation of this paper. This work is supported by the National Natural Science Foundation of China under grant nos. 61231013 and 61271195, the National Science Foundation for Innovative Research Groups of China under grant no. 61221061, the Program for New Century Excellent Talents in University, and the Fundamental Research Funds for the Central Universities.

\section{Author details}

'School of Electronics and Information Engineering, Beihang University, Beijing 100191, China. ${ }^{2}$ Xiamen Air Traffic Management Station, CAAC, Xiamen 361006, China.

Received: 28 July 2013 Accepted: 1 November 2013 Published: 13 November 2013

\section{References}

1. S Moh, C Yu, A cooperative diversity-based robust MAC protocol in wireless ad hoc networks. IEEE Trans. Parallel Distrib. Syst. 22(3), 353-363 (2011)

2. Y Zhou, J Liu, L Zheng, C Zhai, H Chen, Link-utility-based cooperative MAC protocol for wireless multi-hop networks. IEEE Trans. Wireless Commun. 10(3), 995-1005 (2011)

3. C-Y Oh, T-J Lee, Cooperative MAC protocol using active relays for multi-rate WLANs. J Commun Netw. 13(5), 463-471 (2011)

4. H Shan, HT Cheng, W Zhuang, Cross-layer cooperative MAC protocol in distributed wireless networks. IEEE Trans. Wireless Commun. 10(8), 2603-2615 (2011)

5. K-W Chin, Pairwise: a time hopping medium access control protocol for wireless sensor networks. IEEE Trans Consum Electron. 55(4), 1898-1906 (2009) 
6. X Yang, M Peng, J Gibson, GG Xie, D-Z Du, AV Vasilakos, Tight performance bounds of multihop fair access for MAC protocols in wireless sensor networks and underwater sensor networks. IEEE Trans. Mobile Computing. 11(10), 1538-1554 (2012)

7. A Munari, F Rossetto, M Zorzi, Impact of medium access control strategies on the effectiveness of advanced cooperative hybrid ARQ techniques. IEEE Trans. Wireless Commun. 10(9), 2860-2871 (2011)

8. P Liu, Z Tao, S Narayanan, T Korakis, SS Panwar, CoopMAC: a cooperative MAC for wireless LANs. IEEE J. Sel. Areas Commun. 25(2), 340-354 (2007)

9. H Zhu, G Cao, rDCF: a relay-enabled medium access control protocol for ad hoc networks. IEEE Trans. Mobile Comp. 5(9), 1201-1214 (2006)

10. T Guo, RA Carrasco, WL Woo, Differentiated cooperative multiple access for multimedia communications over fading wireless networks. IET Commun. 3(6), 1005-1015 (2009)

11. J-K Lee, H-J Noh, J Lim, A cooperative TDMA MAC protocol using dynamic slot assignment scheme. Paper presented at the international conference on information networking (ICOIN) (Bangkok, Thailand, 2013), pp. 28-30

12. P Liu, C Nie, T Korakis, E Erkip, SS Panwar, F Verde, A Scaglione, STiCMAC: a MAC protocol for robust space-time coding in cooperative wireless LANs. IEEE Trans. Wireless Commun. 11(4), 1358-1369 (2012)

13. M Khalid, Y Wang, I Butun, H Kim, I Ra, R Sankar, Coherence time-based cooperative MAC protocol for wireless ad hoc networks. EURASIP J. Wirel. Commun. Netw. 2011(3), 1-12 (2011)

14. B Cao, G Feng, Y Li, C Wang, Cooperative media access control with optimal relay selection in error-prone wireless networks. IEEE Trans. Veh. Technol. (2012). doi:10.1109/TVT.2012.2226485

15. B Zhou, A Marshall, T-H Lee, A k-round elimination contention scheme for WLANs. IEEE Trans. Mobile Comp. 6(11), 1230-1244 (2007)

16. T Zhou, H Sharif, M Hempel, P Mahasukhon, W Wang, T Ma, A novel adaptive distributed cooperative relaying MAC protocol for vehicular networks. IEEE J. Sel. Areas Commun. 29(1), 72-82 (2011)

17. Y Liu, K Liu, F Zeng, A relay-contention-free cooperative MAC protocol for wireless networks. Paper presented at the IEEE consumer communications and networking conference (CCNC) (Las Vegas, NV, USA, 2011), pp. 9-12

18. PJ Ju, W Song, DZ Zhou, An enhanced cooperative MAC protocol based on perceptron training. Paper presented at IEEE wireless communications and networking conference (WCNC) (Shanghai, China, 2013), pp. 7-10

19. S Sayed, Y Yang, H Hu, CARD: cooperative access with relay's data for multi-rate wireless local area networks. Paper presented at the IEEE international conference on communications (ICC) (Dresden, Germany, 2009), pp. 14-18

20. SG Sayed, Y Yang, J Xu, BTAC: a busy tone based cooperative MAC protocol for wireless local area networks. Mobile Netw. Appl. 16(1), 4-16 (2011)

21. B Escrig, DMT optimal cooperative protocols with destination-based selection of the best relay. IEEE Trans. Wireless Commun. 10(7), 2218-2227 (2011)

22. H Adam, E Yanmaz, C Bettstetter, Medium access with adaptive relay selection in cooperative wireless networks. IEEE Trans. Mobile Comp. (2013). doi:10.1109/TMC.2013.97

23. A Argyriou, Coordinating interfering transmissions in cooperative wireless LANs. IEEE Trans. Wireless Commun. 10(11), 3804-3812 (2011)

24. MG Jibukumar, R Datta, PK Biswas, CoopMACA: a cooperative MAC protocol using packet aggregation. Wireless Netw. 16(7), 1865-1883 (2010)

25. T Aguilar, S-J Syue, V Gauthier, H Afifi, C-L Wang, CoopGeo: a beaconless geographic cross-layer protocol for cooperative wireless ad hoc networks. IEEE Trans. Wireless Commun. 10(8), 2554-2565 (2011)

26. IEEE, Wireless LAN Medium Access Control (MAC) and Physical Layer (PHY) Specifications, IEEE Std. 802.11-2007, Revision of IEEE Std. 802.11-1999 (IEEE, Piscataway, USA, 2007)

doi:10.1186/1687-1499-2013-264

Cite this article as: Li et al:: A rapid cooperation-differentiated medium access control protocol with packet piggyback for multihop wireless networks. EURASIP Journal on Wireless Communications and Networking 2013 2013:264

\section{Submit your manuscript to a SpringerOpen ${ }^{\circ}$ journal and benefit from:}

- Convenient online submission

- Rigorous peer review

- Immediate publication on acceptance

- Open access: articles freely available online

- High visibility within the field

- Retaining the copyright to your article

Submit your next manuscript at $\gg$ springeropen.com 